\title{
Nanoscale Imaging of Structural and Optical Properties Using Helium Temperature Scanning Transmission Electron Microscopy Cathodoluminescence of Nitride Based Nanostructures
}

Frank Bertram ${ }^{1}$, Marcus Müller ${ }^{1}$, Gordon Schmidt ${ }^{1}$, Peter Veit $^{1}$, Silke Petzold $^{1}$, Steven Albert $^{2}$, Ana María Bengoechea-Encabo ${ }^{2}$, Miguel Ángel Sánchez-Garcia², Enrique Calleja ${ }^{2}$, and Jürgen Christen ${ }^{1}$.

1. Institute of Experimental Physics, Otto-von-Guericke-University Magdeburg, Germany.

2. ISOM and Departamento de Ingeniería Electrónica, Universidad Politécnica de Madrid, Spain.

We present the direct nanoscale correlation of structural and optical properties of InGaN/GaN core-shell microrods using highly-spatially resolved cathodoluminescence spectroscopy (CL) in a scanning transmission electron microscope.

For a comprehensive understanding of complex semiconductor heterostructures and the physics of devices based on them, a systematic determination and correlation of the structural, chemical, electronic, and optical properties on a nanometer scale is essential. Luminescence techniques belong to the most sensitive, non-destructive methods of semiconductor research. The combination of luminescence spectroscopy - in particular at liquid He temperatures - with the high spatial resolution of a scanning transmission electron microscopy (STEM) as realized by the technique of low temperature cathodoluminescence microscopy in a STEM (STEM-CL), provides a unique, extremely powerful tool for the optical nano-characterization of quantum structures. Our CL-detection unit is integrated in a FEI STEM Tecnai F20 equipped with a liquid helium stage $(\mathrm{T}=10 \mathrm{~K} / 300 \mathrm{~K})$ and a light collecting parabolic mirror. Panchromatic as well as spectrally resolved (grating monochromator) CL imaging is used. In CL-imaging mode the CL-signal is collected simultaneously to the STEM signal at each pixel. The TEM acceleration voltage is optimized to minimize sample damage and to prevent luminescence degeneration under electron beam excitation.

The core-shell microrod sample under investigation has been grown by a combination of top-down and bottom-up processes. In a first step, regularly ordered $\mathrm{GaN}$ micropillars were obtained by inductively coupled plasma etching of a GaN/sapphire template. Subsequently, the GaN micropillars were overgrown with GaN using plasma assisted molecular beam epitaxy. Finally, a thick InGaN layer was deposited on the whole structure. Scanning electron microscope measurements of the sample reveal a homogeneous array of hexagonally shaped microrods with a density of $1.2 \times 10^{7} \mathrm{~cm}^{-2}$. The mean diameter of the microrods could be estimated to $1.3 \mu \mathrm{m}$.

The cross-sectional STEM image in annular dark field contrast (ADF) of the microrods clearly shows an InGaN capped region of about $750 \mathrm{~nm}$ thickness with a low crystal quality directly on top of the GaN microrod. In addition, we observe a thin InGaN shell layer on the side facets of the microrod with a thickness gradient from $70 \mathrm{~nm}$ to $140 \mathrm{~nm}$ towards the microrod topside. Structural investigations reveal the presence of threading dislocations propagating from the $\mathrm{GaN}$ template through the microrods towards the apex.

Direct comparison of the ADF image of a single microrod with the panchromatic CL intensity image at $16 \mathrm{~K}$ reveals the highest $\mathrm{CL}$ intensity coming from the $\mathrm{InGaN}$ on top as well as on the side walls of the 


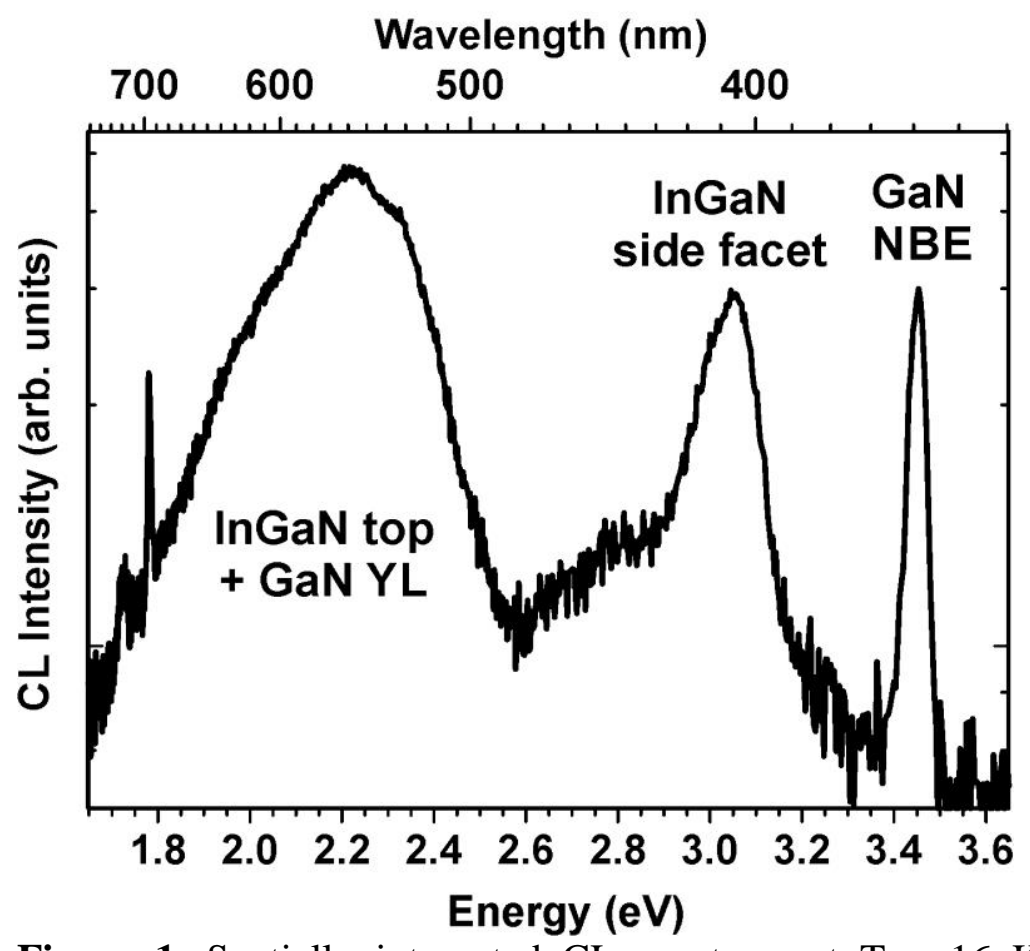

Figure 1. Spatially integrated $\mathrm{CL}$ spectrum at $\mathrm{T}=16 \mathrm{~K}$ exhibits a near band edge emission of $\mathrm{GaN}$, as well as two broad InGaN luminescence contributions. The side walls emit at $3.05 \mathrm{eV}$ whereas the InGaN top shows emission at $2.21 \mathrm{eV}$.
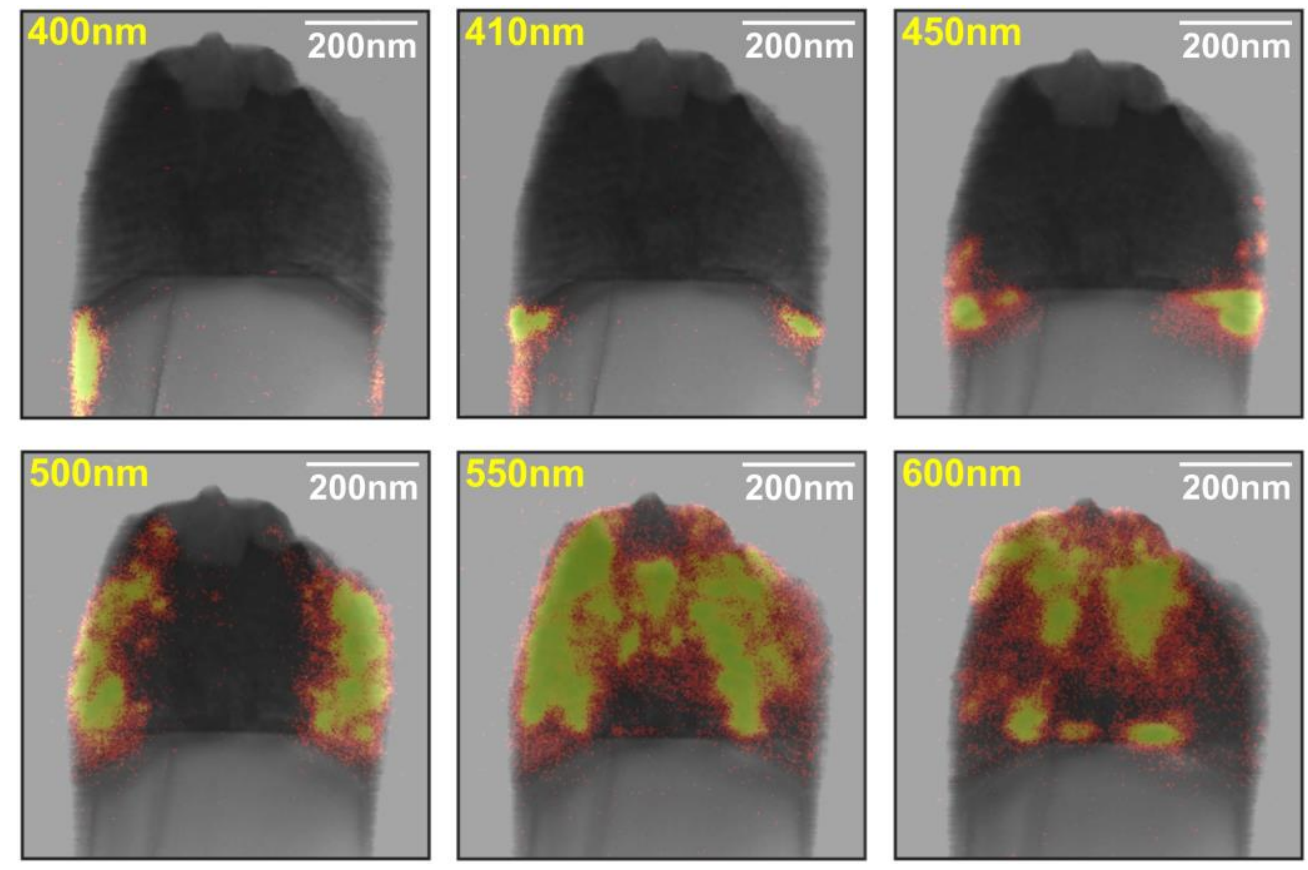

microrod. The integral CL spectrum from a single $\mathrm{InGaN} / \mathrm{GaN}$ microrod exhibits three distinct luminescence contributions: the near band edge emission (NBE) of $\mathrm{GaN}$ and two InGaN peaks. The distribution of the peak wavelength exhibits a dominating NBE emission at $359 \mathrm{~nm}$ $(3.45 \mathrm{eV})$ as well as the yellow defect band at $560 \mathrm{~nm}(2.21 \mathrm{eV})$ out of the center of the microrod. On the side facets one can observe the high energetic InGaN CL at $407 \mathrm{~nm}$ $(3.05 \mathrm{eV})$.

In contrast, the cap layer emits a broad luminescence band between 510 $640 \mathrm{~nm} \quad(2.43-1.94 \mathrm{eV}) . \quad$ This difference of the InGaN emission on the side facet compared to the cap can be attributed to different InGaN compositions and/or strain conditions. Furthermore, a red shift of the InGaN luminescence in both $\mathrm{InGaN}$ regions is observed indicating a gradual increase of the In concentration during the growth due to the lattice pulling effect. 